\title{
Mathematical Methods for Modeling Chemical Reaction Networks
}

\author{
Justin Carden - Jr-PSOC Member, Casian Pantea, Gheorghe Craciun, \\ Raghu Machiraju, Parag Mallick- PSOC Member

$$
\begin{gathered}
E+S 1 \rightleftharpoons E S 1 \quad E+S 2 \rightleftharpoons E S 2 \\
S 2+E S 1 \rightleftharpoons E S 1 S 2 \rightleftharpoons S 1+E S 2 \\
E S 1 S 2 \rightarrow E+P
\end{gathered}
$$

Network (1) includes seven species $E, S 1, S 2, E S 1, E S 2$, $E S 1 S 2, P$ and nine reactions among them (four reversible pairs and one irreversible reaction), whose rate constants are denoted by $k_{1}, \ldots, k_{9}$. The corresponding mass-action system of ODEs is given in (2), and is obtained by collecting in the time derivative of each species the contribution of all reactions to the concentration of that species. For example, $E$ is being produced in reaction $E S 1 \rightarrow E+S 1$; this reaction has rate $k_{2} c_{E S 1}$ and we include this term in the expression of $d c_{E} / d t$ (the time derivative of concentration $c_{E}$ of $E$ ). On the other hand, $E$ is consumed in the reaction $E+S 1 \rightarrow$ $E S 1$, therefore the rate $k_{1} c_{E} c_{S 1}$ of this reaction is added with negative sign in the expression of $d c_{E} / d t$. Note that reactions not involving $E$ (e.g. $S 2+E S 1 \rightarrow E S 1 S 2$ ) do not affect the concentration $c_{E}$, and do not contribute terms to the expression of $d c_{E} / d t$. We assume additionally that substrates $S 1$ and $S 2$ are being supplied to the reaction environment at constant rates $F_{S 1}$ and $F_{S 2}$, and we include these inflow (supply) rates into the expressions of $d c_{S 1} / d t$ and $d c_{S 2} / d t$ respectively. (In mass-action models, the inflow rates are always positive constants.) Moreover, the substrates $S 1, S 2$ and the product $P$ are also being transported out of the reaction environment (or degraded) at rates $\xi_{S 1} c_{S 1}, \xi_{S 2} c_{S 2}$ and $\xi_{P} c_{P}$ proportional to their concentrations (this is always the form of outflow rates in mass-action models, and the positive constants $\xi_{S 1}, \xi_{S 2}$ and $\xi_{P}$ are called outflow coefficients). The outflow terms are included with negative signs in the expressions of $d c_{S 1} / d t, d c_{S 2} / d t$ and $d c_{P} / d t$ respectively.

The most common way to construct ODEs from reaction networks is via the law of mass-action kinetics, under which the rate of a reaction is proportional to the concentration of each reactant ${ }^{1}$ (the proportionality factor is a positive number called the rate constant). Every reaction network has a corresponding mass-action ODE system. To illustrate, consider reaction network (1) of an overall reaction $S 1+S 2 \rightarrow P$, under a mechanism for enzyme catalysis corresponding to unordered substrate binding ${ }^{2}[1]$ :

\footnotetext{
${ }^{1}$ To be exact, proportional to a power of the reactant concentration given by its stoichiometry. For example, the rate of a dimerization $2 A \rightarrow B$ is $k c_{A}^{2}$.

2 This mechanism is involved in cyclin-dependent kinase-catalyzed phosphorylation reactions that play important roles in the cell cycle [2].
}

Research Supported by NIH/NCI PSOC, CF, SJMPFF. P.M. Corresponding Author, phone: 650-724-0923; (paragm@stanford.edu) J.C. Stanford University ( jecarden@stanford.edu). C. P., W Virginia University (pantea@math.wvu.edu). G. $\quad$ C., University of Wisconsin (craciun@math.wisc.edu). $\quad$ R.M. Ohio State University (machiraju.1@osu.edu)

$$
\begin{aligned}
\frac{d c_{E}}{d t} & =-k_{1} c_{E} c_{S 1}+k_{2} c_{E S 1}-k_{3} c_{E} c_{S 2}+k_{4} c_{E S 2} \\
& +k_{9} c_{E S 1 S 2} \\
\frac{d c_{S 1}}{d t} & =-k_{1} c_{E} c_{S 1}+k_{2} c_{E S 1}-k_{7} c_{S 1} c_{E S 2}+k_{8} c_{E S 1 S 2} \\
& +F_{S 1}-\xi_{S 1} c_{S 1} \\
\frac{d c_{S 2}}{d t} & =-k_{3} c_{E} c_{S 2}+k_{4} c_{E S 2}-k_{5} c_{S 2} c_{E S 1}+k_{6} c_{E S 1 S 2} \\
& +F_{S 2}-\xi_{S 2} c_{S 2} \\
\frac{d c_{E S 1}}{d t} & =k_{1} c_{E} c_{S 1}-k_{2} c_{E S 1}-k_{5} c_{E S 1} c_{S 2}+k_{6} c_{E S 1 S 2} \\
\frac{d c_{E S 2}}{d t} & =k_{3} c_{E} c_{S 2}-k_{4} c_{E S 2}-k_{7} c_{E S 2} c_{S 1}+k_{8} c_{E S 1 S 2} \\
\frac{d c_{E S 1 S 2}}{d t} & =k_{5} c_{S 2} c_{E S 1}+k_{7} c_{E S 2} c_{S 1}-\left(k_{6}+k_{8}+k_{9}\right) c_{E S 1 S 2} \\
\frac{d c_{P}}{d t} & =k_{9} c_{E S 1 S 2}-\xi_{P} c_{P}
\end{aligned}
$$


The differential equations (2) depend on 14 parameters (rate constants $k_{1}, \ldots, k_{9}$ and the inflow/outflow rates $\left.F_{S 1}, F_{S 2}, \xi_{S 1}, \xi_{S 2}, \xi_{P}\right)$. The presence of nonlinear terms in such a system warrants the possibility for complex behavior; indeed, it turns out that some choices of parameters ${ }^{3}$ give rise to bistability, i.e. there are two distinct steady states, both compatible with the same amount of enzyme (for example, such that $\left.c_{E}+c_{E S 1}+c_{E S 2}+c_{E S 1 S 2}=1\right)$. For the particular choice of parameters, there are two stable steady states (depicted as red dots in Fig. 1) and one unstable steady state (green dot in Fig.1).

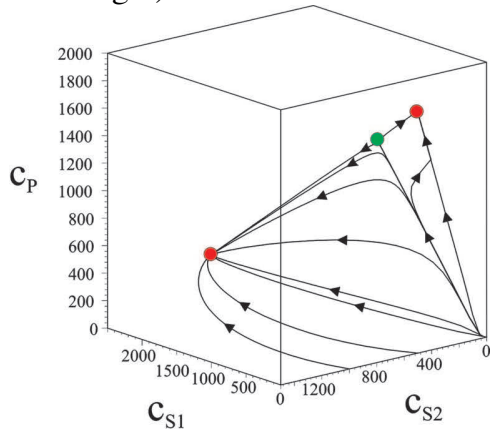

Figure 1. Examples of trajectories for S1, S2, P concentrations. Figure taken from [1].

The two stable steady states are reached from initial concentrations in their respective basins of attraction, and switching between them is possible, for example, by a temporary disturbance in a substrate supply rate.

Numerical simulation may reveal bistability of a network for given values of parameters (rate constants, inflow/outflow rates, enzyme total concentrations). In practice however, there are often no good estimates for parameter values, and sampling a vast parameter space in search of interesting behavior is almost an impossible task. However, in many cases, one can answer questions like "are there parameter values that give rise to bistability for a certain network?" without numerical simulation, but rather based on a body of theoretical work termed reaction network theory.

\section{B. Reaction network theory.}

Beginning with groundbreaking work of Horn, Jackson and Feinberg [3,4,5], reaction network theory addresses the question: "What behaviors of a reaction network are a function of its structure, and are robust to different choices of kinetics?" This question has a natural extension which is practically very important: "How can reaction networks be controlled in order to encourage/rule out certain behaviors?" The early work of Horn, Jackson and Feinberg solved a number of difficult problems on chemical reaction network behavior, for systems with mass-action kinetics.

Recently there has been an explosion of interest in reaction network theory, fueled by the growth of systems

${ }^{3}$ For example, $k_{1}=93.43, k_{2}=2539, k_{3}=481.6, k_{4}=1183, k_{5}=1556, k_{6}=$ $121192, k_{7}=0.02213, k_{8}=1689, k_{9}=85842, \xi_{S 1}=\xi_{S 2}=\xi_{P}=1, F_{S 1}=$ $2500, F_{S 2}=1500$. The values are taken from [1], and they were used to draw Fig. 1. biology, and the development of new and powerful mathematical techniques. A very fruitful area of research has been in identifying conditions for multiple positive steady states in reaction networks $[1,6,7,8,9]$. In particular, the cycle structure of the SR graph [1] or DSR graph [7] associated to a network may rule out bistability for any choice of rate constants. The DSR graph is a directed labeled bipartite graph resembling the schematic of the reaction network. It turns out that the DSR graph of network (1), depicted in Fig.2, cannot rule out the possibility of multiple positive steady states. While this is not enough to insure that bistability is possible, further theory [6] shows that in fact there are choices of parameters for which bistability occurs.

Methods like these show an intimate relationship between network structure and bistability, and provide some surprising and counterintuitive results. For example, Table 1 shows how bistability can be induced by subtle differences in enzyme catalysis mechanisms for the same overall reaction (for example, $S \rightarrow P$ in entries 3 and 4). The webserver CoNtRol [10] implements a series of theoretical methods [8] testing the possibility for bistability in reaction networks. Another powerful software is the Chemical Reaction Network Toolbox [11].

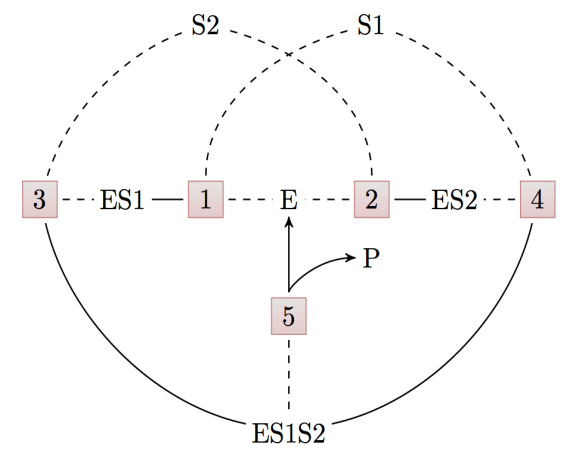

Figure 2. The DSR graph of network (1), generated using the software CoNtRol [10]

Other questions in reaction network theory have also witnessed notable progress: oscillations [12,13]; global stability and global convergence, i.e., informally speaking, the question of when all initial conditions lead to the same stable behavior [14-18]; persistence, i.e., whether some reactant concentrations can decrease to zero despite positive initial concentrations $[14,15,19,20]$.

TABLE 1. Examples of enzymatic networks and their capacity for bistability. Table taken from [1].

Entry
\begin{tabular}{|l|l|l|l|}
\hline 1 & $\mathrm{E}+\mathrm{S} \rightleftarrows \mathrm{ES} \rightarrow \mathrm{E}+\mathrm{P}$ & $\begin{array}{l}\text { Elementary enzyme catalysis } \\
\text { underlying Michaelis-Menten } \\
\text { kinetics: } \mathrm{S} \rightarrow \mathrm{P}\end{array}$ & $\mathrm{N}$ \\
\hline 2 & $\mathrm{E}+\mathrm{S} \rightleftarrows \mathrm{ES} \rightarrow \mathrm{E}+\mathrm{P}, \mathrm{E}+\mathrm{I} \rightleftarrows \mathrm{EI}$ & $\begin{array}{l}\text { Elementary enzyme catalysis with } \\
\text { competitive inhibition: S } \rightarrow \mathrm{P}\end{array}$ & $\mathrm{N}$ \\
\hline 3 & $\mathrm{E}+\mathrm{S} \rightleftarrows \mathrm{ES} \rightarrow \mathrm{E}+\mathrm{P}, \mathrm{ES}+\mathrm{I} \rightleftarrows \mathrm{ESI}$ & $\begin{array}{l}\text { Elementary enzyme catalysis with } \\
\text { uncompetitive inhibition: } \mathrm{S} \rightarrow \mathrm{P}\end{array}$ & $\mathrm{N}$ \\
\hline 4 & $\begin{array}{l}\mathrm{E}+\mathrm{S} \rightleftarrows \mathrm{ES} \rightarrow \mathrm{E}+\mathrm{P}, \mathrm{E}+\mathrm{I} \rightleftarrows \mathrm{EI} \\
\mathrm{ES}+\mathrm{I} \rightleftarrows \mathrm{ESI} \rightleftarrows \mathrm{El}+\mathrm{S}\end{array}$ & $\begin{array}{l}\text { Elementary enzyme catalysis with } \\
\text { mixed inhibition: S } \rightarrow \mathrm{P}\end{array}$ & $\mathrm{Y}$ \\
\hline 5 & $\begin{array}{l}\mathrm{E}+\mathrm{S} 1 \rightleftarrows \mathrm{ES} 1 \\
\mathrm{~S} 2+\mathrm{ES} 1 \rightleftarrows \mathrm{ES} 1 \mathrm{~S} 2 \rightarrow \mathrm{E}+\mathrm{P}\end{array}$ & $\begin{array}{l}\text { Two-substrate enzyme catalysis } \\
\text { with ordered substrate-binding } \\
\text { S1+S2 } \rightarrow \mathrm{P}\end{array}$ & $\mathrm{Y}$ \\
\hline 6 & $\begin{array}{l}\mathrm{E}+\mathrm{S} 1 \rightleftarrows \mathrm{ES} 1 \mathrm{E}+\mathrm{S} 2 \rightleftarrows \mathrm{ES} 2 \\
\mathrm{~S} 2+\mathrm{ES} 1 \rightleftarrows \mathrm{ES} 1 \mathrm{~S} 2 \rightarrow \mathrm{S} 1+\mathrm{ES} 2 \\
\mathrm{ES} 1 \mathrm{~S} 2 \rightarrow \mathrm{E}+\mathrm{P}\end{array}$ & $\begin{array}{l}\text { Two-substrate enzyme catalysis } \\
\text { with un-ordered substrate-binding } \\
\text { S1+S2 } \rightarrow \mathrm{P}\end{array}$ & $\mathrm{Y}$ \\
\hline
\end{tabular}




\section{REFERENCES}

[1] G. Craciun, Y. Tang, and M. Feinberg. Understanding bistability in complex enzyme-driven reaction networks. PNAS 2006103 (23) 86978702

[2] P. M. Clare, R. A. Poorman, L.C. Kelley, K.D. Watenpaugh, B.A. Bannow, K.L. Leach, (2001) J. Biol. Chem. 276, 48292-48299.

[3] F. Horn, R. Jackson, General mass action kinetics, Archive for Rational Mechanics and Analysis 47, 1972.

[4] M. Feinberg, Complex balancing in general kinetic systems, Archive for Rational Mechanics and Analysis 49:3, 1972.

[5] M. Feinberg, Lectures on chemical reaction networks. Notes of lectures given at the Mathematics Research Center of the University of Wisconsin in 1979, http://www.che.eng.ohiostate.edu/ FEINBERG/LecturesOnReactionNetworks.

[6] G. Craciun, M. Feinberg, Multiple equilibria in complex chemical reaction networks: I. the injectivity property, SIAM J. Appl. Math 65:5, 2005.

[7] M. Banaji, G. Craciun, Graph-theoretic approaches to injectivity and multiple equilibria in systems of interacting elements,

Communications in Mathematical Sciences 7:4, 2009.

[8] M. Banaji, C. Pantea, Some results on injectivity and multistationarity in chemical reaction networks. arXiv:1309.6771, 2015.

[9] Angeli D, Ferrell JE Jr, Sontag ED. Detection of multistability, bifurcations, and hysteresis in a large class of biological positivefeedback systems. Proc Natl Acad Sci USA. 101, 2004.

[10] Pete Donnell, Murad Banaji, Anca Marginean, Casian Pantea, CoNtRol: an open source framework for the analysis of chemical reaction networks, Bioinformatics 30, 2014.

[11] P. Ellison, M. Feinberg, H. Ji, Chemical Reaction Network Toolbox, available for download at http://www.crnt.osu.edu/CRNTWin

[12] M. Mincheva and M.R. Roussel. Graph-theoretic methods for the analysis of chemical and biochemical networks. I. Multistability and oscillations in ordinary differential equation models. J. Math. Biol., 55:61-86, 2007.

[13] D. Angeli, M. Banaji, C. Pantea, Combinatorial approaches to Hopf bifurcations in systems of interacting elements, Communications in Mathematical Sciences 12, 2014.

[14] C. Pantea, On the persistence and global stability of mass-action systems, SIAM J. Math. Anal. 44:3, 2012.

[15] G. Craciun, F. Nazarov, C. Pantea, Persistence and permanence of mass-action and power-law dynamical systems, SIAM J. Appl. Math. $73,2013$.

[16] G. Craciun, A. Dickenstein, A. Shiu, B. Sturmfels, Toric Dynamical Systems, Journal of Symbolic Computation 44:11, 2009.

[17] D. F. Anderson, A proof of the Global Attractor Conjecture in the single linkage class case, SIAM J. Appl. Math., 71:4, 2011.

[18] Manoj Gopalkrishnan, Ezra Miller and Anne Shiu. A geometric approach to the global attractor conjecture SIAM J. Appl. Dyn. Syst., 13:2, 2014

[19] D. Angeli, P. De Leenheer, E. D. Sontag, A petri net approach to the study of persistence in chemical reaction networks, Math Biosci, 210:2, 598-618, 2007.

[20] [97] A. Shiu and B. Sturmfels, Siphons in chemical reaction networks, Bull. Math. Biol., 72 (2010), pp. 1448-1463. 